All authors: Radboud university medical center, Nijmegen, the Netherlands.

Published online ahead of print at www.jco.org on July 13, 2015

Supported by Pink Ribbon, the Netherlands (Grant No. 2009-2013)

Presented at the International PsychoOncology Society 15th World Congress of Psycho-Oncology, Rotterdam, the Netherlands, November 6-8, 2013; and at the 13th International Congress of Behavioral Medicine, Groningen, the Netherlands, August 20-23, 2014.

The study sponsor had no role in the study design, data collection, analysis and interpretation of data, writing of the report, or decision to submit the paper for publication. The authors had full access to all data and had final responsibility for the decision to submit for publication.

Authors' disclosures of potential conflicts of interest are found in the article online at www.jco.org. Author contributions are found at the end of this article.

Corresponding author: Sanne W. van den Berg, MSc, Radboud university medical center, Department of Medical Psychology (840), PO Box 9101, 6500 HB Nijmegen, the Netherlands; e-mail: sanne.vandenberg@radboudumc.nl.

(c) 2015 by American Society of Clinica Oncology

0732-183X/15/3325w-2763w/\$20.00 DOI: $10.1200 / J C O .2013 .54 .9386$

\title{
BREATH: Web-Based Self-Management for Psychological Adjustment After Primary Breast Cancer-Results of a Multicenter Randomized Controlled Trial
}

Sanne W. van den Berg, Marieke F.M. Gielissen, José A.E. Custers, Winette T.A. van der Graaf, Petronella B. Ottevanger, and Judith B. Prins

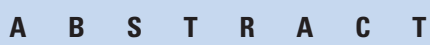

\section{Purpose}

Early breast cancer survivors (BCSs) report high unmet care needs, and easily accessible care is not routinely available for this growing population. The Breast Cancer E-Health (BREATH) trial is a Web-based self-management intervention to support the psychological adjustment of women after primary treatment, by reducing distress and improving empowerment.

\section{Patients and Methods}

This multicenter, randomized, controlled, parallel-group trial evaluated whether care as usual (CAU) plus BREATH is superior to CAU alone. BREATH is delivered in sixteen fully automated weekly modules covering early survivorship issues. Two to 4 months post-treatment, BCSs were randomly assigned to receive CAU + BREATH $(n=70)$ or CAU alone $(n=80)$ using a stratified block design (ratio 1:1). Primary outcomes were distress (Symptom Checklist-90) and empowerment (Cancer Empowerment Questionnaire), assessed before random assignment (baseline, T0) and after 4 (T1), 6 (T2), and 10 months (T3) of follow-up. Statistical (analysis of covariance) and clinical effects (reliable change index) were tested in an intention-to-treat analysis (T0 to T1). Follow-up effects (TO to T3) were assessed in assessment completers.

\section{Results}

CAU + BREATH participants reported significantly less distress than CAU-alone participants $(-7.79 ; 95 \% \mathrm{Cl},-14.31$ to $-1.27 ; P=.02)$ with a small-to-medium effect size $(d=0.33)$, but empowerment was not affected $(-1.71 ; 95 \% \mathrm{Cl}, 5.20$ to $-1.79 ; P=.34)$. More CAU + BREATH participants (39 of 70 [56\%]; $95 \%$ Cl, 44.1 to 66.8) than CAU-alone participants (32 of 80 [40\%]; $95 \% \mathrm{Cl}, 30.0$ to 51.0$)$ showed clinically significant improvement $(P=.03)$. This clinical effect was most prominent in low-distress BCSs. Secondary outcomes confirmed primary outcomes. There were no between-group differences in primary outcomes during follow-up.

\section{Conclusion}

Access to BREATH reduced distress among BCSs, but this effect was not sustained during follow-up.

\section{J Clin Oncol 33:2763-2771. (C) 2015 by American Society of Clinical Oncology}

\section{INTRODUCTION}

Women diagnosed with breast cancer face challenges that do not end with treatment completion. The first year after primary treatment, the so-called re-entry phase, ${ }^{1}$ is characterized by physical, emotional, and social recovery. ${ }^{2}$ Women report high unmet care needs ${ }^{3}$ and have to cope with lingering physical and emotional symptoms of treatment, fear of recurrence, decreasing social support, losing the safety net of care providers, and resuming professional and recreational activities. ${ }^{2,4-6}$ Approximately $70 \%$ of breast cancer survivors (BCSs; ie, women who have completed primary breast cancer treat- ment with no evidence of recurrence ${ }^{7}$ ) adjust well during the re-entry phase, but a substantial proportion report high levels of distress. ${ }^{8,9}$

Better diagnosis and therapy mean that more women survive breast cancer, and self-management strategies $^{10}$ and e-health ${ }^{11}$ have been proposed as ways to support this growing population. Because approximately half of all BCSs already search the Internet for breast cancer-specific information, ${ }^{12-15}$ the Internet is promising for providing psychooncological interventions. ${ }^{16}$ However, there are few randomized controlled trials (RCTs) of Web-based interventions to support re-entry adjustment. To date, most Web-based interventions including 


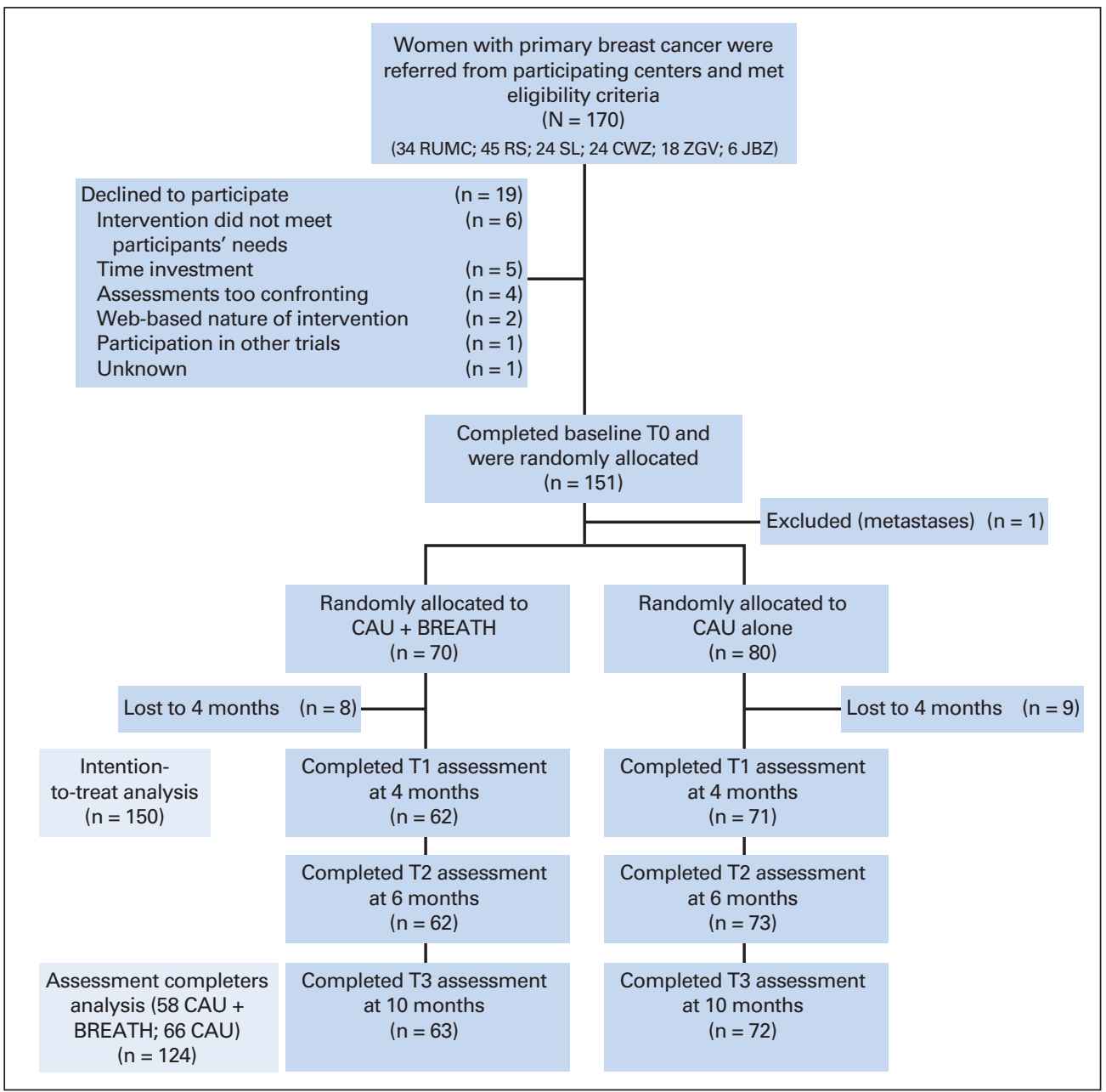

Fig 1. Trial profile. BREATH, Breast Cancer E-Health intervention; CAU, care as usual; CWZ, Canisius-Wilhelmina Hospital, Nijmegen; JBZ, Jeroen Bosch Hospital, Den Bosch; RS, Rijnstate Hospital, Arnhem and Zevenaar; RUMC, Radboud university medical center, Nijmegen; SL, Slingeland Hospital, Doetinchem; ZGV, Hospital Gelderse Vallei, Ede.

women with breast cancer have not been either re-entry specific ${ }^{17-20}$ or breast cancer specific, ${ }^{21,22}$ or have predominantly focused on peer support groups ${ }^{23-26}$ or informational support. ${ }^{27}$ In a recent overview advocating re-entry specific care, Stanton ${ }^{2}$ reported promising but inconclusive evidence from seven RCTs that psychoeducational interventions can be effective in BCSs.

We developed the Breast Cancer E-Health (BREATH) trial $^{28}$ a Web-based self-management intervention on the basis of cognitive behavioral therapy that provides early BCSs with self-management skills to enable them to take control of, and adjust to, post-treatment survivorship. The intervention was developed using the transactional model of stress ${ }^{29}$ and the model of psychological well-being in cancer survivors, ${ }^{30}$ both of which describe negative and positive outcomes in adapting to a stressor. Thus, the primary aim of this RCT was to study whether care as usual (CAU) plus BREATH (CAU + BREATH) can effectively target negative and positive adjustment. We hypothesized that CAU + BREATH is superior to CAU alone in reducing distress and improving empowerment. Because BCSs exhibit different levels of distress during the re-entry phase, subhypotheses were that CAU + BREATH would reduce distress in high-distress BCSs, keep levels of distress low in low-distress BCSs, and improve empowerment in both distress groups.

\section{PATIENTS AND METHODS}

\section{Study Design and Participants}

The BREATH study protocol has been published elsewhere. ${ }^{28}$ We conducted a multicenter, randomized, controlled, parallelgroup trial to evaluate the efficacy of a Web-based selfmanagement intervention in facilitating psychological adjustment among BCSs. One university hospital and five regional hospitals in the Netherlands participated (Fig 1). Female BSCs were eligible if they had a histologically proven malignancy of the breast and had completed curative-intent primary treatment (defined as surgery plus adjuvant chemotherapy and/or radiotherapy) 2 to 4 months before the baseline assessment. Participant characteristics are listed in Table 1 .

The local treatment team monitored patient recruitment and eligibility and obtained informed consent. A researcher (S.v.d.B.) contacted participants to check additional eligibility criteria: understanding the Dutch language, access to the Internet, and having an e-mail address

Study assessments covered the first year after breast cancer, with baseline at 2 to 4 months after completion of primary treatment (T0), and follow-up assessments at 4 (T1), 6 (T2), and 10 (T3) months after 


\begin{tabular}{|c|c|c|c|c|}
\hline \multirow[b]{2}{*}{ Characteristic } & \multicolumn{2}{|c|}{$\begin{array}{c}\text { CAU + } \\
\text { BREATH } \\
(n=70)\end{array}$} & \multicolumn{2}{|c|}{$\begin{array}{l}\text { CAU alone } \\
(\mathrm{n}=80)\end{array}$} \\
\hline & $\begin{array}{c}\text { No. of } \\
\text { Patients }\end{array}$ & $\%$ & $\begin{array}{c}\text { No. of } \\
\text { Patients }\end{array}$ & $\%$ \\
\hline \multicolumn{5}{|l|}{ Age, years } \\
\hline Mean & \multicolumn{2}{|c|}{51.44} & \multicolumn{2}{|c|}{50.18} \\
\hline SD & \multicolumn{2}{|l|}{8.30} & \multicolumn{2}{|c|}{9.15} \\
\hline \multicolumn{5}{|l|}{ Educational level* } \\
\hline Low (ISCED 0-1-2) & 14 & 20 & 13 & 16 \\
\hline Medium (ISCED 3-4-5) & 32 & 46 & 48 & 60 \\
\hline High (ISCED 6-7-8) & 24 & 34 & 19 & 24 \\
\hline \multicolumn{5}{|l|}{ Marital status } \\
\hline Married/cohabiting & 58 & 83 & 61 & 76 \\
\hline Unmarried & 5 & 7 & 5 & 6 \\
\hline Divorced & 5 & 7 & 10 & 13 \\
\hline Widowed & 2 & 3 & 4 & 5 \\
\hline Children (yes) & 61 & 87 & 62 & 76 \\
\hline \multicolumn{5}{|l|}{ Employment† } \\
\hline Paid work outside home & 30 & 43 & 32 & 40 \\
\hline Home management & 21 & 30 & 18 & 23 \\
\hline Unemployed & 3 & 4 & 8 & 10 \\
\hline Sick leave & 30 & 43 & 42 & 53 \\
\hline Disability insurance act & 3 & 4 & 5 & 6 \\
\hline Voluntary work & 7 & 10 & 4 & 5 \\
\hline Student & 0 & 0 & 1 & 1 \\
\hline Retired & 3 & 4 & 3 & 4 \\
\hline \multicolumn{5}{|l|}{ Treatment type } \\
\hline Surgery + chemotherapy + radiotherapy & 48 & 69 & 56 & 70 \\
\hline Surgery + chemotherapy & 19 & 27 & 22 & 28 \\
\hline Surgery + radiotherapy & 3 & 4 & 2 & 2 \\
\hline Hormone therapy & 46 & 66 & 53 & 66 \\
\hline Low distress $(\mathrm{GSI} \leq 0.57)$ & 51 & 73 & 55 & 69 \\
\hline \multicolumn{5}{|l|}{ Frequency of Internet use $\neq$} \\
\hline Daily & 24 & 59 & 38 & 73 \\
\hline 2 to 4 times a week & 12 & 29 & 9 & 17 \\
\hline Weekly or less & 5 & 12 & 5 & 10 \\
\hline \multicolumn{5}{|c|}{$\begin{array}{l}\text { NOTE. No significant differences were found between the two conditions } \\
(P>.05) \text {. P values were calculated using } \chi^{2} \text { tests for categorical variables and } \\
\text { a two-tailed Students's } t \text { test (independent samples) for continuous variables. } \\
\text { Abbreviations: BREATH, Breast Cancer E-Health intervention; CAU, care as } \\
\text { usual; GSI, General Severity Index; ISCED, International Standard Classifica- } \\
\text { tion of Education; SD, standard deviation. } \\
\text { *ISCED } 2011 \text {. } \\
\text { †Percentages do not add up to } 100 \% \text { because more options are possible. } \\
\text { fCAU + BREATH }(\mathrm{n}=41) \text {, CAU alone }(\mathrm{n}=52) \text {. }\end{array}$} \\
\hline
\end{tabular}

baseline. The Radboud University Medical Center Medical Review Ethics Committee (file No. 2009/144) and the ethics boards of local participating centers approved the study (Netherlands Trial Register NTR2935).

\section{Intervention}

BREATH (Appendix Fig A1, online only) targets re-entry issues relevant to BCSs during a fixed 16-week modular program for four phases of adjustment to breast cancer (looking back, emotional processing, strengthening, and looking ahead). ${ }^{28}$ Intervention components (104 total) are based on cognitive behavioral therapy and include information (26 scripts), assignment (48 tasks, or homework), assessment (10 self-tests followed by automated feedback), and video (20 topics illustrated with clips extracted from interviews). BREATH is a pure self-help program without therapist contact (also known as self-administered therapy ${ }^{31}$ ). Each week, new materials are released, accompanied by standard e-mail reminders, in an attempt to maintain or improve adherence. Access to BREATH was withdrawn after 16 weeks.

BCSs receiving CAU alone did not have access to BREATH. For both conditions, CAU consisted of visits to an oncologist three times per month and psychosocial care on demand or referral. No restrictions were made regarding use of Internet or psychological or other self-help interventions.

\section{Outcomes}

Study assessments and random assignment were conducted online using RadQuest software (Radboudumc Medical Psychology, Nijmegen, the Netherlands). Baseline characteristics were obtained with a medical checklist derived from the patient chart.

General psychological distress was assessed with the Symptom Checklist-90 (SCL-90; range, 90-450), which has good psychometric properties in healthy and patient populations. ${ }^{32}$ The SCL-90 total score showed good internal consistency at baseline (Cronbach's $\alpha=$ .97). For the General Severity Index (GSI), which represents the mean score of all responses, transformed item scores (0-4) were used. General psychological empowerment was assessed with the Cancer Empowerment Questionnaire (CEQ). ${ }^{33}$ The CEQ presumes that patients can derive strength from themselves (intrapersonal) and from their social surroundings (interpersonal). Baseline internal consistency was good (Cronbach's $\alpha=.92$ ).

Secondary outcomes reflected negative adjustment (general ${ }^{34,35}$ and cancer-specific distress, ${ }^{36}$ fatigue, ${ }^{37}$ helplessness, ${ }^{38}$ and fear of cancer recurrence ${ }^{39,40}$ ) and positive adjustment (self-efficacy, ${ }^{41}$ remoralization, ${ }^{42}$ personal control, ${ }^{43}$ quality of life, ${ }^{44}$ fulfillment, ${ }^{45}$ reevaluation, ${ }^{45}$ new ways of living, ${ }^{45}$ and valuing life $\left.{ }^{45}\right)$. For details of secondary outcomes, see our study protocol ${ }^{28}$ (or the legend of Table 2 with treatment effects).

Information about self-reported use of Internet and other resources (individual support, peer support, rehabilitation support groups) was collected at T1. General Internet use was assessed with the question "Have you consulted the Internet for information on (learning to live with) breast cancer in the past 4 months?" Four-month usage data of CAU + BREATH participants were evaluated. ${ }^{46}$ For the current report, correlations were calculated between the mean difference ( $\mathrm{T} 0$ to $\mathrm{T} 1$ ) in distress and the continuous usage variables of frequency of log-ins, total duration (in minutes), and activity (number of intervention components opened).

\section{Random Assignment and Masking}

BCSs were randomly assigned (allocation ratio 1:1) to receive $\mathrm{CAU}+\mathrm{BREATH}$ or CAU alone. For each center, a randomized block design with stratification by hormone therapy was generated. After baseline assessment, a random number generator with variable block sizes of 4,6 , and 8 automatically ensured blinded allocation until intervention assignment. One researcher (S.v.d.B.) informed participants about treatment assignment by e-mail and was therefore not blinded. In one case, a participant was told an incorrect treatment assignment (CAU instead of CAU + BREATH); this participant received CAU alone and her data were analyzed accordingly. 


\section{Statistical Methods}

All statistical analyses were performed with SPSS 20 (IBM, Armonk, NY). The sample size calculation for the primary outcomes at T1 was 170: 128 BCSs ( 64 in each group) at 80\% power for differences between CAU + BREATH and CAU alone with a medium effect size of 0.50 , plus a $25 \%$ dropout rate. ${ }^{28}$ The level of significance in the sample size calculation was adjusted to $P \leq .025$ to keep the overall chance of type I error at $5 \%$. Inclusion was prematurely stopped at 151 participants, with approval of the ethics committee, because only $5 \%$ (7 of 151) of the participants dropped out at T1.

\section{Statistical Effect}

The primary hypothesis was that CAU + BREATH would be superior to CAU alone in decreasing distress and increasing empowerment, and it was tested in an intention-to-treat (ITT) analysis of data for T0 and T1. Missing data at T1 were imputed using last observation carried forward. The significance of intervention effects on primary and secondary outcomes was tested using one-way between-groups analyses of covariance with group (CAU + BREATH or CAU alone) as a fixed factor. Because participants were not preselected before inclusion, the analysis of covariance model corrected for baseline differences. For primary outcome analyses, baseline distress (SCL-90) and empowerment (CEQ) were used as covariates. For secondary outcome analyses, baseline scores of corresponding questionnaires were used as covariates. Interaction effects of mean adjusted differences between $\mathrm{T} 0$ and $\mathrm{T} 1$ by group are reported in Table 2, with SEs, significance level, and 95\% CI. Effect size Cohen's $d$ for independent groups was calculated using the pooled standard deviations and unadjusted means on $\mathrm{T} 1$.

\section{Clinically Significant Change}

Clinically significant change, assessed with the reliable change index (RCI) of the GSI, was tested in ITT analysis (T0 to T1). Following Jacobson and Truax, ${ }^{47}$ Schauenburg and Strack ${ }^{48}$ calculated the GSI cutoff 0.567 to discriminate between low and high distress on the basis of normative and psychotherapy samples. The magnitude of improvement (defined as RCI $\leq-0.16$ for lowdistress participants and RCI $\leq-0.43$ for high-distress participants) or deterioration (RCI $\geq 0.16$ for low-distress participants and RCI $\geq 0.43$ for high-distress participants) was assessed using one-sided $\chi^{2}$ tests. $^{33}$

\section{Follow-Up Effect}

Follow-up effects for primary and secondary outcomes were evaluated with mixed within-between repeated-measures analysis of variance, including data for participants who completed all four assessments (T0 to T3). Because participants were randomly assigned at each center, center was not included as a random effect. Baseline variables were taken into account as within factors in the model. Differences between CAU + BREATH and CAU alone were tested using independent samples $t$ tests.

\section{RESULTS}

Between August 2010 and March 2012, 170 women were referred and 151 (89\%) underwent random assignment (Fig 1). Data collection was finalized in February 2013. One woman was errone- ously enrolled (metastatic disease) and excluded after the random assignment, ${ }^{49}$ leaving a final ITT sample of $150 \mathrm{BCSs}$ (70 CAU + BREATH, $80 \mathrm{CAU}$ alone). At baseline, the two groups did not differ on demographic characteristics (Table 1) and study outcomes (Table 2). Participants with missing data at T1 $(n=17)$ had higher levels of baseline distress than participants with complete data at T1 (mean difference, $23.57 ; 95 \% \mathrm{CI}, 3.82$ to $43.31 ; P=.02$ ). Levels of baseline empowerment were similar $(P=.79)$. Missing data at $\mathrm{T} 1$ were equally distributed between the two groups. Overall, 124 participants (58 CAU + BREATH; 66 CAU alone) completed all four assessments, and their data were included in follow-up analyses. No metastases or severe illnesses were reported during the study. One woman was admitted to a psychiatric clinic; this was reported as a serious adverse event.

\section{Statistical Effect}

The decrease in distress at $\mathrm{T} 1$ was significantly greater in CAU + BREATH participants than in CAU-alone participants, with a small-to-medium effect size $(d=0.33$; Table 2$)$. Baseline distress explained $53 \%$ of the variance in distress at $\mathrm{T} 1(P<.005)$. No such difference in empowerment was found.

Secondary outcome analyses (Table 2) revealed that CAU+ BREATH led to significant improvements in five of seven negative adjustment variables (general and cancer-specific distress, fatigue, and two fear of cancer recurrence outcomes) with small-to-medium effect sizes ( $d=0.37$ to 0.55 ), and in 3 of 10 positive adjustment variables (self-efficacy, remoralization, new ways of living) with small-tomedium effect sizes ( $d=0.26$ to 0.39 ).

\section{Clinically Significant Change}

More CAU + BREATH participants (39 of 70 [56\%]; 95\% CI, 44.1 to 66.8 ) than CAU-alone participants (32 of 80 [40\%]; 95\% CI, 30.0 to 51.0$)$ showed a clinically significant improvement $(P=.03)$. We had hypothesized that more high-distress BCSs would show a clinically significant improvement after CAU + BREATH than after CAU alone, but this was not the case ( 10 of 21 [48\%]; 95\% CI, 28.3 to $67.6 v 14$ of 27 [52\%]; $95 \%$ CI, 34.0 to 69.3 , respectively; $P=.39$ ). Post hoc analysis revealed that there was no difference in the proportion of high-distress BCSs showing clinical deterioration (5 of 21 [24\%] $v 2$ of 27 [7\%], respectively; $P=.06$ ). Of the low-distress BCSs, more CAU + BREATH participants than CAU-alone participants showed clinical improvement or no change (41 of 49 [84\%]; $95 \%$ CI, 71.0 to $91.5 v 35$ of 53 [66\%]; $95 \%$ CI, 52.6 to 77.3 respectively; $P=.02$ ). Moreover, explorative post hoc analyses of low-distress BCSs revealed that, compared with CAU-alone participants, CAU + BREATH participants showed more clinically significant improvement (29 of 49 [59\%] $v 18$ of 53 [34\%], respectively; $P=.006$ ) and less deterioration ( 8 of 49 [16\%] $v 18$ of 53 [34\%], respectively; $P=.02$ ). The empowerment hypothesis was not tested, because empowerment was not significantly different between CAU + BREATH and CAU alone.

\section{Follow-Up Effect}

At T2 and T3, distress was significantly reduced regardless of group assignment $(\mathrm{F}[3,120]=5.88 ; P=.001 ; \mathrm{Fig} 2)$. This was also true for the secondary negative adjustment outcomes of fear of cancer recurrence (Cancer Worry Scale; $\mathrm{F}[3,120]=5.954 ; P=.001$ ), fatigue $(\mathrm{F}[3,120]=4.40 ; P=.006)$, and helplessness $(\mathrm{F}[3,120]=11.964$; $P=.000)$. A significant time $\times$ group interaction effect was found for 


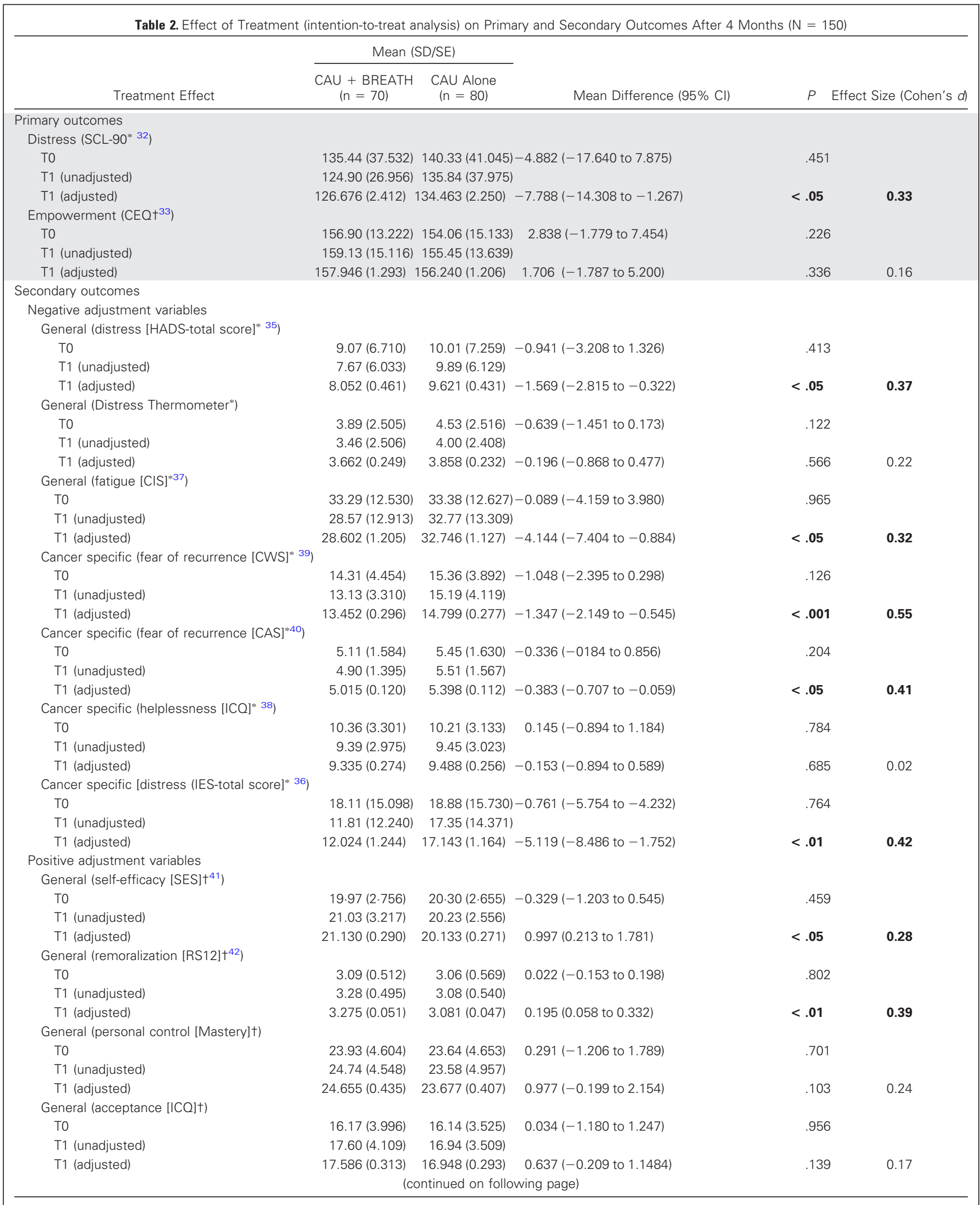




\begin{tabular}{|c|c|c|c|c|c|}
\hline \multirow[b]{2}{*}{ Treatment Effect } & \multicolumn{2}{|c|}{ Mean (SD/SE) } & \multirow[b]{2}{*}{ Mean Difference $(95 \% \mathrm{Cl})$} & \multirow[b]{2}{*}{$P$} & \multirow[b]{2}{*}{ Effect Size (Cohen's $d$} \\
\hline & $\begin{array}{c}\text { CAU + BREATH } \\
(\mathrm{n}=70)\end{array}$ & $\begin{array}{l}\text { CAU Alone } \\
(n=80)\end{array}$ & & & \\
\hline \multicolumn{6}{|l|}{ General (perceived benefits [ICO]†) } \\
\hline TO & $15.09(4.169)$ & $15.86(3.801)$ & $-0.777(-2.063$ to 0.509$)$ & .235 & \\
\hline T1 (unadjusted) & $15.66(4.584)$ & $16.21(4.541)$ & & & \\
\hline T1 (adjusted) & $16.042(0.363)$ & $15.926(0.339)$ & $0.116(-0.866$ to 1.098$)$ & .816 & 0.12 \\
\hline \multicolumn{6}{|c|}{ Cancer specific (quality of life [EORTC QLQ-C30] ${ }^{40}$ ) } \\
\hline T0 & $66.79(16.575)$ & $69.79(17.906)$ & $-3.006(-8.601$ to 2.589$)$ & .290 & \\
\hline T1 (unadjusted) & $72.50(18.572)$ & $70.52(15.231)$ & & & \\
\hline T1 (adjusted) & $73.295(1.784)$ & $69.882(1.666)$ & $3.413(-1.411$ to 8.236$)$ & .164 & 0.12 \\
\hline \multicolumn{6}{|l|}{ Cancer specific (fulfillment $[\mathrm{PAO}] \mathrm{\dagger}^{45}$ ) } \\
\hline TO & $38.30(6.686)$ & $39.80(6.934)$ & $-1.500(-3.706$ to 0.706$)$ & .181 & \\
\hline T1 (unadjusted) & 40.89 (6.728) & $40.10(7.289)$ & & & \\
\hline T1 (adjusted) & $41.284(0.759)$ & $39.788(0.708)$ & $1.496(-0.55$ to 3.548$)$ & .152 & 0.11 \\
\hline \multicolumn{6}{|c|}{ Cancer specific (re-evaluation [PAQ]†) } \\
\hline TO & $38.39(6.160)$ & $40.01(6.407)$ & $-1.627(-3.662$ to 0.409$)$ & .116 & \\
\hline T1 (unadjusted) & $38.81(6.493)$ & $39.44(7.107)$ & & & \\
\hline T1 (adjusted) & $39.380(0.611)$ & $38.835(0.570)$ & $0.545(-1.107$ to 2.197$)$ & .516 & 0.09 \\
\hline \multicolumn{6}{|c|}{ Cancer specific (new ways of living [PAQ]†) } \\
\hline TO & $41.19(5.279)$ & $41.34(7.488)$ & $-0.152(-2.271$ to 1.967$)$ & .888 & \\
\hline T1 (unadjusted) & $42.94(6.230)$ & $41.07(8.012)$ & & & \\
\hline T1 (adjusted) & $43.001(0.709)$ & $41.032(0.663)$ & 1.969 (0.051 to 3.886$)$ & $<.05$ & 0.26 \\
\hline \multicolumn{6}{|l|}{ Cancer specific (valuing life [PAQ]†) } \\
\hline TO & $34.06(5.778)$ & $34.51(4.963)$ & $-0.455(-2.188$ to 1.278$)$ & .604 & \\
\hline T1 (unadjusted) & $34.40(6.087)$ & $34.11(5.972)$ & & & \\
\hline T1 (adjusted) & $34.595(0.545)$ & $33.971(0.510)$ & $0.623(-0.853$ to 2.100$)$ & .405 & 0.05 \\
\hline $\begin{array}{l}\text { NOTE. All } P \text { values were calculated us } \\
\text { (Adjusted) means adjusted for baseline } \\
\text { Abbreviations: BREATH, Breast Cancer } \\
\text { Checklist Individual Strength; CWS, } \\
\text { Questionnaire Core } 30 \text { items; HADS, } \\
\text { Adjustment Questionnaire; RS12, Rem } \\
\text { "Increase represents worsening; decre } \\
\text { tIncrease represents improvement; }\end{array}$ & $\begin{array}{l}\text { ovariance with adju } \\
\text { onding questionnai } \\
\text { n; CAS, Cancer Acc } \\
\text { EORTC QLQ-C30, } \\
\text { Depression Scale; } \\
\text { tems; SCL-90, Sym } \\
\text { rovement. } \\
\text { vorsening. }\end{array}$ & $\begin{array}{l}\text { stment for baseline } \\
\text { re. Bold font indica } \\
\text { eptance Scale; CAL } \\
\text { European Organi } \\
\text { ICQ, Illness Cogn } \\
\text { ptom Checklist-90 }\end{array}$ & $\begin{array}{l}\text { value. (Unadjusted) means } \\
\text { tes significant effect and cor } \\
\text {, care as usual; CEQ, Cancer } \\
\text { sation for Research and Tre } \\
\text { ition Questionnaire; IES, Imp } \\
\text { items; SD, standard deviation }\end{array}$ & $\begin{array}{l}\text { unadjustec } \\
\text { respondin } \\
\text { Empower } \\
\text { atment of } \\
\text { pact of Eve } \\
\text { 7; SES, Se }\end{array}$ & $\begin{array}{l}\text { d for baseline covariates. } \\
g \text { effect size. } \\
\text { ment Questionnaire; CIS, } \\
\text { f Cancer Quality of Life } \\
\text { ent Scale; PAQ, Positive } \\
\text { If-Efficacy Scale. }\end{array}$ \\
\hline
\end{tabular}

fear of cancer recurrence (Cancer Worry Scale; $\mathrm{F}[3,120]=4.563 ; P=$ $.005)$, with CAU + BREATH participants reporting less fear than CAU-alone participants at T2 $(-1.459 ; 95 \% \mathrm{CI},-2.743$ to -0.175$)$. Of the positive adjustment outcomes, acceptance significantly improved in both groups (ICQ; F[3, 120] $=8.531 ; P=.000$ ). Time effects and time $X$ group interactions were not significant for all remaining outcomes, including empowerment (Fig 3).

\section{Use of BREATH and Other Resources}

Use of the BREATH intervention varied considerably. Frequency of logins ranged from 0 to 45 , total duration ranged from 0 to 2,324 minutes, and activity ranged from 0 to 104 intervention components opened. The mean difference in distress (SCL-90, T0 to T1) was not correlated with frequency $(r=-0.007 ; P=.96)$, total duration $(r=$ $0.000 ; P=1.00)$, or activity $(r=-1.072 ; P=.55)$.

At T1, similar proportions of women in CAU + BREATH and CAU alone had consulted the Internet in the previous 4 months on a monthly (24\% v 34\%), weekly (13\% v 8\%), or daily ( $0 \% v 2 \%)$ basis, or not at all $(61 \% v 58 \%)$. There were also no significant differences ( $\mathrm{n}=126 ; P=.27$ ) between CAU + BREATH and CAU-alone participants in the use of individual support (eg, psychologist; $12 \% v 25 \%$, respectively), peer and rehabilitation support groups (14\% v12\%, respectively), or combined individual and group support (21\% v13\%, respectively). Half of the participants in both groups did not make use of other support (53\% $v 50 \%$, respectively).

\section{DISCUSSION}

At the start of the re-entry phase, 4-month access to BREATH in addition to CAU resulted in a statistically and clinically significant distress reduction compared with CAU alone. However, this small-tomedium effect was not sustained, and levels of distress were similar at 6 and 10 months. CAU + BREATH participants also showed a greater decrease than CAU-alone participants in fear of cancer recurrence, fatigue, and general and cancer-related distress. The effect of BREATH on fear of cancer recurrence was sustained during follow-up. Access to BREATH did not influence empowerment or clinical distress improvement in high-distress BCSs. Low-distress BCSs showed a greater clinical improvement and less deterioration with CAU + BREATH than with CAU alone.

The RCT was designed according to quality standards (CONSORT for parallel group, ${ }^{50}$ nonpharmacologic treatment, ${ }^{51}$ and eHealth trials). ${ }^{52,53}$ Statistically and clinically significant changes were evaluated in ITT analyses, with missing data imputed using a conservative method (last observation carried forward) 


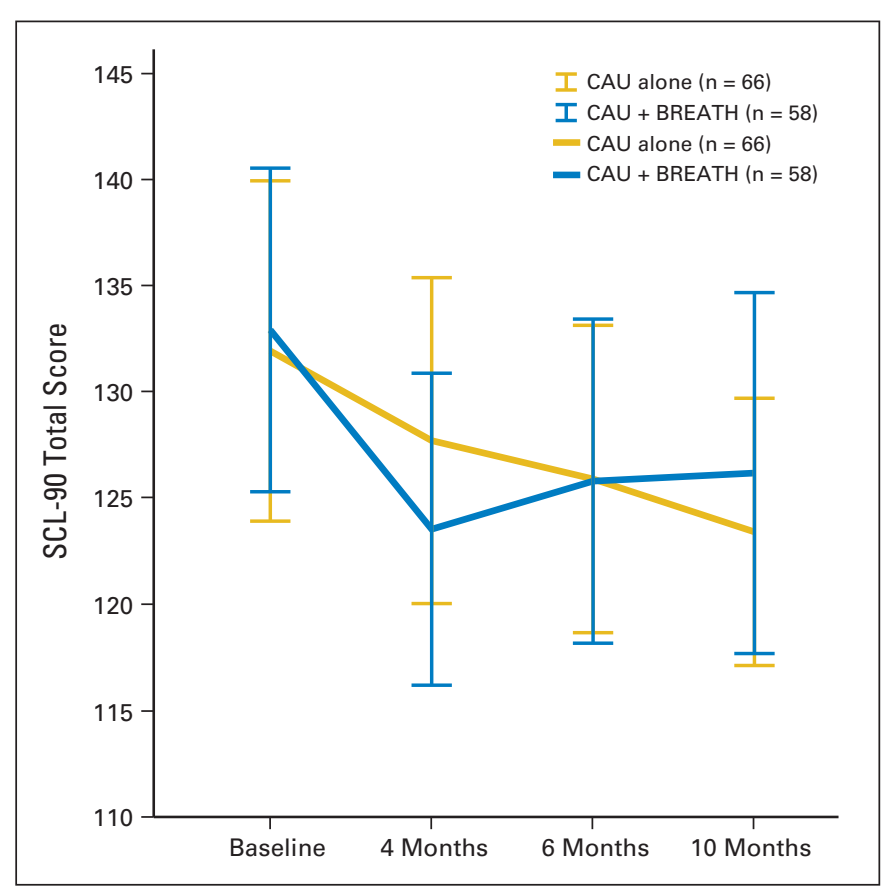

Fig 2. Psychological distress (Symptom Checklist-90 [SCL-90]) at baseline and 4, 6, and 10 months after baseline ( $n=124$ assessment completers). Vertical bars represent the $95 \% \mathrm{Cl}$ of the mean SCL-90 at each time point. BREATH, Breast Cancer E-Health intervention;; CAU, care as usual.

because the patients in our sample were expected to improve on distress over the study period. ${ }^{8,9}$ The multicenter recruitment strategy guaranteed referrals from both secondary and tertiary care centers. The recruitment procedure, which ensured minimal involvement of the research team, and lack of assistance regarding intervention use and adherence support the ecological validity of BREATH. In the absence of RCTs evaluating similar unguided Web-based interventions for BCSs, the effect size of BREATH is consistent with that of two recent meta-analyses of guided face-toface (effect size range 0.26 to 0.38 ) $^{54}$ and Web-based (effect size range, 0.17 to 0.21$)^{55}$ interventions for people with cancer ${ }^{54}$ and chronic somatic conditions. $^{55}$

The secondary outcomes revealed that CAU + BREATH decreased general and cancer-specific distress, fatigue, and fear of cancer recurrence, which may reflect the multicomponent nature of distress in cancer patients. ${ }^{56}$ The clinical relevance of these outcomes needs to be addressed in future research.

CAU + BREATH did not significantly change empowerment relative to the effect of CAU alone and had inconsistent effects on the secondary positive adjustment variables. The study of positive adjustment is a new research area and poses multiple challenges. Although new models of survivorship care stress patient empowerment, ${ }^{57,58}$ there is no consensus about the empowerment construct. Consequently, positive adjustment questionnaires, such as the CEQ, are new, but not extensively validated in BCSs and lack information on sensitivity to change. Furthermore, in psycho-oncology, resourceoriented therapeutic models ${ }^{59}$ are lacking, and interventions are traditionally aimed at diminishing deficits instead of enhancing strengths. It is possible that BREATH does not include a sufficient number of empowerment modules (only 4 of the 16 weekly modules targeted empowerment).

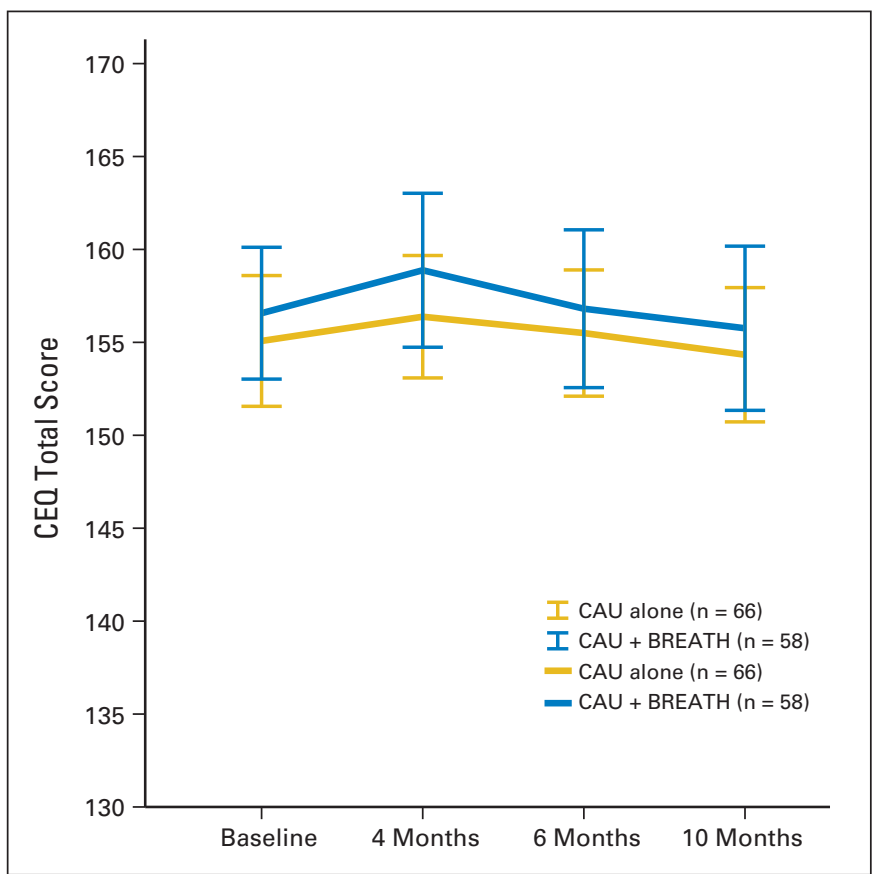

Fig 3. Psychological empowerment (Cancer Empowerment Questionnaire [CEQ]) at baseline and 4, 6, and 10 months after baseline ( $n=124$ assessment completers). Vertical bars represent the $95 \% \mathrm{Cl}$ of the mean $\mathrm{CEQ}$ at each time point. BREATH, Breast Cancer E-Health intervention; CAU, care as usual.

Results should be considered with caution for several reasons. Consistent with the scarce literature on Web-based interventions for cancer patients, ${ }^{60}$ BREATH did not have a sustained effect on distress. This may be because access to the Web site was for 4 months only. Although the limited access enabled accurate postintervention assessments, in retrospect it might have been better to allow participants to retain access, especially because information often remains available with other psychoeducational interventions or self-help books. Another explanation for lack of a sustained effect may be the small-to-medium effect size, which might not have been enough to compensate for the natural course of emotional recovery. The missing-at-random assumption for imputation was violated. BCSs with missing data for the 4-month assessment had significantly higher levels of distress at baseline. Although not significant, more high-distress BCSs in the CAU + BREATH group showed a clinical deterioration. This leads to a cautious interpretation of the results regarding high-distress BCSs, and suggests that these women may need a more intensive intervention than BREATH.

The limited data on BREATH use means it is not possible to draw firm conclusions about how often the intervention should be used to have an effect. Further investigations with larger samples, mediation analyses, or usage pattern are needed to gain insight into determinants of intervention use and to study a possible doseresponse relationship. ${ }^{61}$ Data for BCSs who declined to participate were not recorded. Although it was not feasible to recruit patients consecutively, our sample seemed homogeneous and representative, because the mean age, treatment type, education, and work situation of participants were comparable to those of other studies evaluating the Dutch breast cancer population. ${ }^{3,9,62,63}$ The study sample also proved representative with regard to psychological 
functioning during the first year after treatment. As in other studies of patients with breast ${ }^{8,9}$ or other ${ }^{64}$ cancer, most patients experienced low levels of distress ( $n=102[68 \%])$.

To the best of our knowledge, this is the first RCT to demonstrate an additional effect of a self-management intervention specifically designed to support BCSs in the year after treatment completion. Although small, the primary effect on distress was statistically robust and clinically relevant. Moreover, the intervention does not necessarily require a lot of user commitment. Future research should focus on replicating the current findings, using more valid questionnaires for the positive adjustment variables, and evaluating the follow-up effect beyond 4 months of access. The magnitude of the effect in BCSs with low and high distress should be investigated further. BREATH demonstrated its potential as a feasible first step in a matched supportive care model providing evidence-based and easily accessible re-entry care.
AUTHORS' DISCLOSURES OF POTENTIAL CONFLICTS OF INTEREST

Disclosures provided by the authors are available with this article at www.jco.org.

\section{AUTHOR CONTRIBUTIONS}

Conception and design: Sanne W. van den Berg, Petronella B.

Ottevanger, Judith B. Prins

Administrative support: Sanne W. van den Berg

Provision of study materials or patients: Petronella B. Ottevanger

Collection and assembly of data: Sanne W. van den Berg

Data analysis and interpretation: All authors

Manuscript writing: All authors

Final approval of manuscript: All authors

\section{REFERENCES}

1. Mullan F: Re-Entry: The educational needs of the cancer survivor. Health Educ Q 10 (suppl):88-94, 1984

2. Stanton AL: What happens now? Psychosocial care for cancer survivors after medical treatment completion. J Clin Oncol 30:1215-1220, 2012

3. Pauwels E, Charlier C, De Bourdeaudhuij I, et al: Care needs after primary breast cancer treatment: Survivors' associated sociodemographic and medical characteristics. Psycho-Oncology 22:125132, 2013

4. Allen JD, Savadatti S, Levy AG: The transition from breast cancer 'patient' to 'survivor.' PsychoOncology 18:71-78, 2009

5. Arnold EM: The cessation of cancer treatment as a crisis. Soc Work Health Care 29:21-38, 1999

6. Ganz PA, Kwan L, Stanton AL, et al: Physical and psychosocial recovery in the year after primary treatment of breast cancer. J Clin Oncol 29:11011109, 2011

7. Bell K, Ristovski-Slijepcevic S: Cancer survivorship: Why labels matter. J Clin Oncol 31:409-411, 2013

8. Helgeson VS, Snyder P, Seltman H: Psychological and physical adjustment to breast cancer over 4 years: Identifying distinct trajectories of change. Health Psychol 23:3-15, 2004

9. Henselmans I, Helgeson VS, Seltman $\mathrm{H}$, et al: Identification and prediction of distress trajectories in the first year after a breast cancer diagnosis. Health Psychol 29:160-168, 2010

10. McCorkle R, Ercolano E, Lazenby $M$, et al: Self-management: Enabling and empowering patients living with cancer as a chronic illness. CACancer J Clin 61:50-62, 2011

11. Aaronson NK, Mattioli $V$, Minton $O$, et al: Beyond treatment - Psychosocial and behavioural issues in cancer survivorship research and practice. Eur J Cancer Supplements 12:54-64, 2014

12. Afshari M, Ghani MFA, Radzi NM: Use of the Internet by breast cancer patients. Sci Res Essays 6:3571-3576, 2011

13. Eysenbach G: The impact of the Internet on cancer outcomes. CA-Cancer J Clin 53:356-371, 2003

14. Fogel J, Albert SM, Schnabel F, et al: Use of the Internet by women with breast cancer. J Med Internet Res 4:e9, 2002
15. Littlechild SA, Barr L: Using the Internet for information about breast cancer: A questionnairebased study. Patient Educ Couns, 2013

16. Leykin $Y$, Thekdi SM, Shumay DM, et al: Internet interventions for improving psychological well-being in psycho-oncology: Review and recommendations. Psycho-Oncology 21:1016-1025, 2012

17. Berry DL, Hong F, Halpenny B, et al: Electronic self-report assessment for cancer and selfcare support: Results of a multicenter randomized trial. J Clin Oncol 32:199-205, 2014

18. Carpenter KM, Stoner SA, Schmitz K, et al: An online stress management workbook for breast cancer. J Behav Med, 2012

19. Stanton AL, Thompson EH, Crespi CM, et al: Project Connect Online: Randomized trial of an Internet-based program to chronicle the cancer experience and facilitate communication. J Clin Oncol 31:3411-3417, 2013

20. Ventura F, Ohlen J, Koinberg I: An integrative review of supportive e-health programs in cancer care. Eur J Oncol Nurs 17:498-507, 2013

21. Beatty L, Koczwara B, Wade T: 'Cancer Coping Online': A pilot trial of a self-guided CBT Internet intervention for cancer-related distress. Electron $\mathrm{J}$ Appl Psychol 7:17-25, 2011

22. Duffecy J, Sanford S, Wagner L, et al: Project onward: An innovative e-health intervention for cancer survivors. Psycho-Oncology 22:947-951, 2013

23. Gustafson DH, Hawkins R, Pingree S, et al: Effect of computer support on younger women with breast cancer. J Gen Intern Med 16:435-445, 2001

24. Hoybye MT, Dalton SO, Deltour I, et al: Effect of Internet peer-support groups on psychosocial adjustment to cancer: $\mathrm{A}$ randomised study. $\mathrm{Br} \mathrm{J}$ Cancer 102:1348-1354, 2010

25. Owen JE, Klapow JC, Roth DL, et al: Randomized pilot of a self-guided Internet coping group for women with early-stage breast cancer. Ann Behav Med 30:54-64, 2005

26. Lepore $S$, Buzaglo J, Lieberman $M$, et al: Comparing standard versus prosocial Internet support groups for patients with breast cancer: A randomized controlled trial of the helper therapy principle. J Clin Oncol 32:4081-4294, 2014

27. Ryhanen AM, Siekkinen M, Rankinen $S$, et al: The effects of Internet or interactive computerbased patient education in the field of breast cancer: A systematic literature review. Patient Educ Couns 79:5-13, 2010

28. van den Berg SW, Gielissen MF, Ottevanger PB, et al: Rationale of the BREAst cancer e-healTH [BREATH] multicentre randomised controlled trial:
An Internet-based self-management intervention to foster adjustment after curative breast cancer by decreasing distress and increasing empowerment. BMC Cancer 12:394, 2012

29. Lazarus F, Folkman S: Stress, appraisal, and coping. New York, NY, Springer, 1984

30. Andrykowski MA, Lykins E, Floyd A: Psychological health in cancer survivors. Semin Oncol Nursing 24:193-201, 2008

31. Newman MG, Szkodny LE, Llera SJ, et al: A review of technology-assisted self-help and minimal contact therapies for anxiety and depression: Is human contact necessary for therapeutic efficacy? Clin Psychol Rev 31:89-103, 2011

32. Arindell WA, Ettema JHM: Handleiding bij een multidimensionele psychopathologie-indicator: Symptom Checklist SCL-90. (Herziene uitgave). Swets \& Zeitlinger, Lisse, the Netherlands, 2003

33. van den Berg SW, van Amstel FK, Ottevanger $\mathrm{PB}$, et al: The Cancer Empowerment Questionnaire: Psychological empowerment in breast cancer survivors. J Psychosoc Oncol 31:565-583, 2013

34. Tuinman MA, Gazendam-Donofrio SM, HoekstraWeebers JE: Screening and referral for psychosocial distress in oncologic practice: Use of the distress thermometer. Cancer 113:870-878, 2008

35. Vodermaier A, Millman RD: Accuracy of the Hospital Anxiety and Depression Scale as a screening tool in cancer patients: A systematic review and meta-analysis. Support Care Cancer 19:1899-1908, 2011

36. van der Ploeg E, Mooren TT, Kleber RJ, et al: Construct validation of the Dutch version of the Impact of Event Scale. Psychol Assess 16:16-26, 2004

37. Gielissen MFM, Verhagen $S$, Witjes $F$, et al: Effects of cognitive behavior therapy in severely fatigued disease-free cancer patients compared with patients waiting for cognitive behavior therapy: A randomized controlled trial. J Clin Oncol 24:48824887, 2006

38. Evers AWM, Kraaimaat FW, van Lankveld W, et al: Beyond unfavorable thinking: The illness cognition questionnaire for chronic diseases. J Consult Clin Psychol 69:1026-1036, 2001

39. Custers JAE, van den Berg SW, van Laarhoven HWM, et al: Cancer Worry Scale: Detecting fear of cancer recurrence in breast cancer survivors. Cancer Nurs 37:E44-E50, 2014

40. Servaes $P$, Verhagen $S$, Schreuder HW, et al: Fatigue after treatment for malignant and benign bone and soft tissue tumors. J Pain Symptom Manage 26:1113-1122, 2003 
41. Servaes $P$, Verhagen $S$, Bleijenberg G: Determinants of chronic fatigue in disease-free breast cancer patients: A cross-sectional study. Ann Oncol 13:589-598, 2002

42. Vissers W, Keijsers GPJ, van der Veld WM, et al: Development of the Remoralization Scale: An extension of contemporary psychotherapy outcome measurement. Eur J Psychol Assessment 26:293 301, 2010

43. Henselmans I, Sanderman R, Helgeson VS, et al: Personal control over the cure of breast cancer: Adaptiveness, underlying beliefs and correlates. Psycho-Oncology 19:525-534, 2010

44. Aaronson NK, Ahmedzai S, Bergman B, et al: The European Organization for Research and Treatment of Cancer QLQ-C30: A quality-of-life instrument for use in international clinical trials in oncology. J Natl Cancer Inst 85:365-376, 1993

45. Boot JS, Holcombe C, Salmon P: Positive adjustment to breast cancer: Development of a disease-specific measure and comparison of women diagnosed from 2 weeks to 5 years. PsychoOncology 19:1187-1194, 2010

46. van den Berg SW, Peters EJ, Kraaijeveld JF, et al: Usage of a generic Web-based selfmanagement intervention for breast cancer survivors: Substudy analysis of the BREATH trial. J Med Internet Res 15:e170, 2013

47. Jacobson NS, Truax P: Clinical significance: A statistical approach to defining meaningful change in psychotherapy research. J Consult Clin Psychol 59 12-19, 1991
48. Schauenburg H, Strack M: Measuring psychotherapeutic change with the Symptom Checklist SCL 90 R. Psychother Psychosom 68:199-206, 1999

49. Fergusson D, Aaron SD, Guyatt G, et al: Post-randomisation exclusions: The intention to treat principle and excluding patients from analysis. BMJ 325:652-654, 2002

50. Moher D, Hopewell S, Schulz KF, et al: CONSORT 2010 explanation and elaboration: Updated guidelines for reporting parallel group randomised trials. BMJ 340:c332, 2010

51. Boutron I, Moher D, Altman DG, et al: Extending the CONSORT statement to randomized trials of nonpharmacologic treatment: Explanation and elaboration. Ann Intern Med 148:295-309, 2008

52. Baker TB, Gustafson DH, Shaw $B$, et al: Relevance of CONSORT reporting criteria for research on eHealth interventions. Patient Educ Couns 81:S77-S86, 2010

53. Eysenbach G, Grp C-E: CONSORT-EHEALTH: Improving and standardizing evaluation reports of Web-based and mobile health interventions. J Med Internet Res 13:e126, 2011

54. Faller $H$, Schuler $M$, Richard $M$, et al: Effects of psycho-oncologic interventions on emotional distress and quality of life in adult patients with cancer: Systematic review and meta-analysis. J Clin Oncol 31:782-793, 2013

55. van Beugen S: Internet-based cognitive behavioral therapy for patients with chronic somatic conditions: A meta-analytic review. J Med Internet Res 16:e88, 2014
56. Holland JC, Andersen B, Breitbart WS, et al: Distress management. J Natl Compr Canc Netw 8:448485, 2010

57. Hood L, Friend SH: Predictive, personalized, preventive, participatory (P4) cancer medicine. Nat Rev Clin Oncol 8:184-187, 2011

58. Pravettoni G, Gorini A: A P5 cancer medicine approach: Why personalized medicine cannot ignore psychology. J Eval Clin Pract 17:594-596, 2011

59. Priebe S, Omer S, Giacco D, et al: Resourceoriented therapeutic models in psychiatry: Conceptual review. Brit J Psychiat 204:256-261, 2014

60. McAlpine $H$, Joubert $L$, Martin-Sanchez F, et al: A systematic review of types and efficacy of online interventions for cancer patients. Patient Educ Couns 98:283-295, 2015

61. Donkin L, Hickie IB, Christensen $H$, et al: Rethinking the dose-response relationship between usage and outcome in an online intervention for depression: Randomized controlled trial. J Med Internet Res 15:67-80, 2013

62. Hinnen $C$, Ranchor $A V$, Sanderman $R$, et al: Course of distress in breast cancer patients, their partners, and matched control couples. Ann Behav Med 36:141-148, 2008

63. Vos PJ, Visser AP, Garssen B, et al: Effects of delayed psychosocial interventions versus early psychosocial interventions for women with early stage breast cancer. Patient Educ Couns 60:212-219, 2006

64. Dunn J, Ng SK, Breitbart W, et al: Healthrelated quality of life and life satisfaction in colorectal cancer survivors: Trajectories of adjustment. Health Qual Life Outcomes 11:46, 2013 


\section{Acknowledgment}

We thank all patients who gave their time to participate and the oncologists, radiotherapists, and research nurses of the participating hospitals: Radboud university medical center, Nijmegen; Rijnstate, Arnhem and Zevenaar; Slingeland, Doetinchem; Hospital Gelderse Vallei, Ede;

Canisius-Wilhelmina Hospital, Nijmegen; and Jeroen Bosch, Den Bosch.

\section{Appendix}

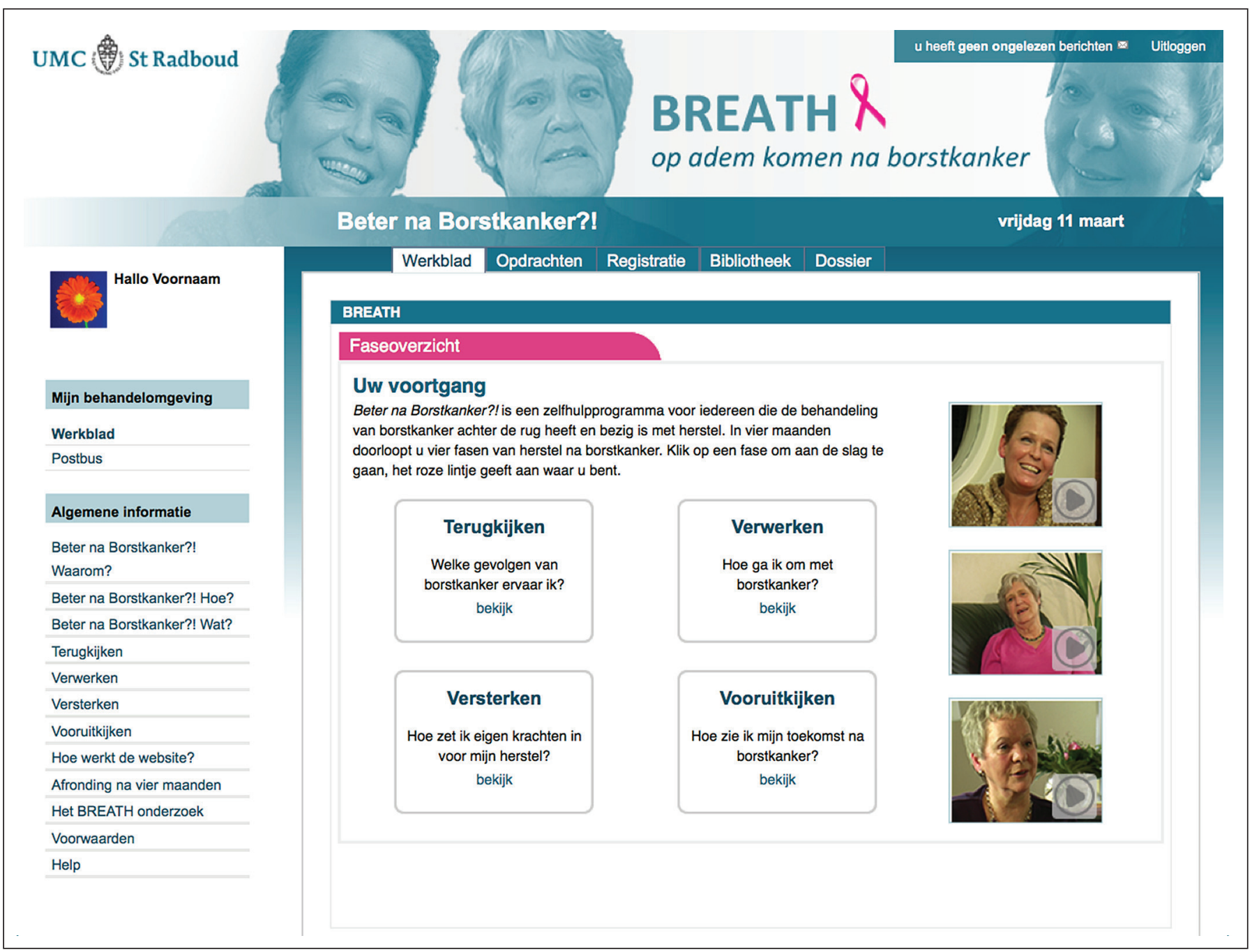

Fig A1. Screenshot of the BREATH (Breast Cancer E-Health intervention) Web site (in Dutch) with four-phase structure. 